\title{
Spin State Read-Out by Quantum Jump Technique: for the Purpose of Quantum
}

\section{Computing}

\author{
E. Pazy Department of Physics, Ben-Gurion University of the Negev, Beer-Sheva 84105, Israel
}

T. Calarco and P. Zoller Institut für Theoretische Physik, Universität Innsbruck, A-6020 Innsbruck, Austria

\begin{abstract}
Utilizing the Pauli-blocking mechanism we show that shining circular polarized light on a singly charged quantum dot induces spin dependent fluorescence. Employing the quantum-jump technique we demonstrate that this resonance luminescence, due to a spin dependent optical excitation, serves as an excellent read out mechanism for measuring the spin state of a single electron confined to a quantum dot.
\end{abstract}

\section{INTRODUCTION}

Semiconductor self-assembled quantum dots (QDs) form spontaneously during the epitaxial growth process, with confinement provided in all three dimensions by a high bandgap in the surrounding material (for example, see [1]). The strong confinement in such structures leads to a discrete atom-like density of states which makes these QDs especially attractive for novel device applications in fields such as quantum computing, optics, and optoelectronics. It is therefore tempting to try to import successful methods used in quantum optics to the field of QDs. As we shall show, one should be cautious in considering the atom-QD analogy. Unlike electrons

\footnotetext{
E.Pazy, acknowledges support through a Kreitman fellowship.
}

in an isolated atom, self-assembled QDs are embedded in an underlying lattice ,i.e., a three-dimensional crystal structure. Even though the number of atoms in such dots is small compared to lithographically defined QDs, still this underlying lattice in which the QD is formed strongly affects the single particle states through its band structure.

Due to their discrete density of states, QDs have been suggested as the major building blocks for numerous quantum computing implementation schemes. These implementation schemes can roughly be divided into those which utilize the charge [2], or respectively the spin [3] of charge carriers confined within a QD. Accurate measurement of a single qubit is an essential requirement for the implementation of quantum computation (QC), therefore highly precise methods for the measurement of the spin of a QD confined charge carrier need to be devised. Implementation of a highly efficient solid-state measurement scheme designed to measure the spin or charge of single electron is an extremely difficult task [5]. An important requirement is that the measurement apparatus do not induce decoherence of qubits while no measurement is taking place. Laser based measurement 
schemes are ideal in this respect since there is no dephasing while the laser field is turned off. There have been proposals for optical measurements of charge using laser pulses [6]. Monitoring the fluorescence from a single quantum dot (QD) has been suggested as a mean to measure single scattering events within QDs [7], as well as a means for final read out of the spin state for the purpose of quantum computation [8]. Recently, it has been verified experimentally that the spin state of an electron residing in a QD can be read using circularly polarized light [9]. In this paper we start with the case with no heavy/light hole mixing, which has also been recently treated in [10], and then we proceed to demonstrate how it is still possible to devise an optical read out scheme even in the presence of heavy/light hole mixing.

Starting with the Hamiltonian for a singly charged QD coupled to the laser field, we consider $\sigma^{+}$-polarized laser pulses in resonance with the lowest excitation. Under these conditions we will show how via the Pauli blocking mechanism, experimentally verified in QDs [11], we are able to obtain spin conditional coupling of a QD-confined electron to the laser field. Restricting the single QD Hamiltonian to a simplified (ideal) three-level scheme and utilizing the quantum jump method we will show how fluorescence can be employed to measure the spin of a single electron residing in the QD. The idea behind this read-out technique is the following: When the polarized laser pulse is switched on, due to Pauliblocking only one of the spin states of the confined electron will optically excite a charged exciton state producing coherent oscillations between the electron-photon and trion state, these being disrupted by spontaneous emission of photons. Therefore, depending on the initial spin state of the electron, in one case the luminescence will exhibit bright periods whereas for the other spin state it will remain dark, i.e. no photons will be emitted from the QD. We will proceed to describe the effects of heavy/light hole mixing on our proposed measurement implementation scheme, showing that although mixing invalidates the assumption of perfect Pauli-blocking it is still possible to use our measurement scheme to read out the spin state of an electron. We also consider the effects of finite detection efficiency.

\section{MODEL}

In the quantum jump technique the dynamics of a quantum system is described by a non-unitary time evolution defined by an effective non-Hermitian Hamiltonian. We start by defining the coherent evolution of a singly occupied QD coupled to a laser field.

The general Hamiltonian describing the dynamics of a single QD interacting with a classical light field can be schematically written as [1]

$$
H=H_{0}+H_{c l}=\left(H_{c}+H_{c c}\right)+H_{c l},
$$

where $H_{c}$ accounts for the non-interacting confined carriers, i.e., electrons and holes, $H_{c c}$ describes the Coulomb interaction between charge carriers and $H_{c l}$ describes the interaction with the classical light field.

\section{A. Single particle states}

To demonstrate the validity of our proposed measurement scheme we employ the traditional approach to obtaining single particle states, utilizing the envelope function approximation [1], [12] combining it with the $\mathbf{k} \cdot \mathbf{p}$ approximation [13], in which the QD wave function are defined in terms of $\Gamma$-like bulk band edge states. Utilizing the above method we classify the single particle states according to the value of $\left(|\mathbf{S}+\mathbf{m}|, S_{z}+m_{z}\right)$, where $S_{z}$ and $m_{z}$ are projections of the spin and internal band angular momentum in the crystal growth direction. We 
obtain the states $(3 / 2, \pm 3 / 2)$ - heavy-hole (HH) subband, the $(3 / 2, \pm 1 / 2)$ states - light-hole (LH) subbandand the $(1 / 2, \pm 1 / 2)$ - spin-orbit split-off subband-the latter of which we can safely ignore being energetically far apart. It is convenient for later calculations of the dipole matrix element to introduce the four $\Gamma$ point Bloch functions [14] which serve as a basis for the crystal states with energies corresponding to the top of the occupied valence band or the bottom of the conduction band. These states are labeled: $|S\rangle,|X\rangle,|Y\rangle,|Z\rangle$. The following are the expressions for the $\mathrm{HH}$ and $\mathrm{LH}$ states in terms of the Bloch functions and the spin of the functions:

$$
\begin{aligned}
& H H:\left\{\begin{aligned}
(3 / 2,3 / 2) & \leftrightarrow \frac{1}{\sqrt{2}}|(X+\imath Y) \uparrow\rangle \\
(3 / 2,-3 / 2) & \leftrightarrow \frac{1}{\sqrt{2}}|(X-\imath Y) \downarrow\rangle
\end{aligned}\right. \\
& L H:\left\{\begin{aligned}
(3 / 2,1 / 2) & \leftrightarrow-\sqrt{\frac{2}{3}}|Z \uparrow\rangle+\frac{1}{\sqrt{6}} \mid(X+\imath Y) \downarrow \\
(3 / 2,-1 / 2) & \leftrightarrow-\sqrt{\frac{2}{3}}|Z \downarrow\rangle-\frac{1}{\sqrt{6}} \mid(X-\imath Y) \uparrow
\end{aligned}\right.
\end{aligned}
$$

Further more we employ the effective mass approximation and strain-induced effects are not treated explicitly, rather, we consider an effective confinement potential.

It is important to stress that our proposed measurement scheme does not relay on the above approach for obtaining the single particle states, we could just as well employed an atomistic psuedo-potential approach[15]. The necessary requirements which need to be satisfied in order to employ our scheme are that single particle states are well separated, energetically, and have well defined angular momentum values.

\section{B. Qubit states}

The states we are interested in measuring via the quantum-jump technique are the spin states of an excess electron confined to a QD. These states serve as qubits for several schemes of quantum computation [3], [4]. We label the spin states of the excess electron with

$$
\begin{aligned}
|0\rangle & \equiv c_{0,-1 / 2}^{\dagger}|\mathrm{vac}\rangle, \\
|1\rangle & \equiv c_{0,1 / 2}^{\dagger}|\mathrm{vac}\rangle,
\end{aligned}
$$

where $c_{0, \sigma}^{\dagger}\left(c_{0, \sigma}\right)$ denote creation (annihilation) operators for electrons in their single-particle ground states with spin projections $\sigma$ and $|\mathrm{vac}\rangle$ stands for the electronhole vacuum, i.e., the crystal ground state. In terms of Bloch functions these states are given by: $|1\rangle \leftrightarrow$ $\imath|S \uparrow\rangle,|0\rangle \leftrightarrow \imath|S \downarrow\rangle$ These are eigenstates of the bare Hamiltonian $H^{c}$, with eigenvalues $\epsilon_{0,-1 / 2}^{e}$ and $\epsilon_{0,1 / 2}^{e}$ respectively, and are not affected by the carrier-carrier interaction $H^{c c}$.

\section{Pauli blocking}

On shining, in the growth direction, a $\sigma^{+}$polarized classical light laser pulse defined by an amplitude $E(t)$ and central frequency $\omega_{L}$ on a QD with an excess electron in the ground state, if the laser is tuned on the lowest interband excitation energy, a ground-state exciton can be obtained. The Pauli exclusion principle forbids double occupancy of any of the electronic states. In particular, if the excess electron occupies the state $|0\rangle$, no further electron can be promoted from the valence band into that state, and thus creation of a charged exciton by a $\sigma^{+}$-polarized laser pulse is inhibited (left part of Fig 1]. On the other hand, if the excess electron was in $|1\rangle$, nothing could prevent a second electron from being excited to the spin down state, thereby creating the charged exciton state (right part of Fig 1). We consider the absolute value of the Rabi frequency, defined as

$$
\Omega(t) \equiv \frac{2 \mu_{00}^{-1 / 2,+3 / 2} E(t)}{\hbar},
$$

(where $\mu_{00}$ denotes the dipole matrix element between the electron and hole ground state wave functions), to be much smaller than the intraband excitation energy, 


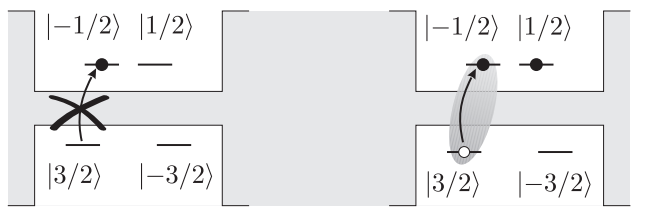

Fig. 1. Pauli-blocking mechanism: a pulse of $\sigma_{+}$-polarized light can promote an electron from the valence band to the conduction-band $-1 / 2$-spin state of a quantum dot only if the latter is not occupied, i.e., if the excess electron in the dot is in the opposite spin state (right). Otherwise, no excitation takes place (left).

$|\Omega| \ll \omega_{e, h}$. Then we can safely neglect the probability of promoting the electron from the valence band to a higher-excited conduction band state. Under these assumptions, the interaction Hamiltonian simplifies to

$$
H_{c l}=\hbar \Omega(t) e^{-i \omega_{L} t} c_{0,-1 / 2}^{\dagger} d_{0,+3 / 2}^{\dagger}+\text { h.c. },
$$

where $d_{0, \sigma}^{\dagger}\left(d_{0, \sigma}\right)$ denote creation (annihilation) operators for holes in their single-particle ground states with spin projections $\sigma$. The spin polarization of the created electron-hole pair $(-1 / 2,3 / 2)$ respectively, is due to the $\sigma^{+}$polarization of the light field. If the temperature is sufficiently low with respect to the electronic intraband excitation energy, $k_{B} T \ll \hbar \omega_{e}$, then we can neglect also the excited states of the excess electron.

\section{Trion state}

Since we will be considering QDs in the strong confinement regime, i.e., the QD level spacing is the largest energy scale in the problem, a good approximation is to assume that the system remains in its ground state. The above consideration allows us to consider a single-QD three-level sub-space scheme: two states which correspond to the two spin states of an excess electron in the QD $(|0\rangle,|1\rangle)$ and the third state is the the so-called "trion" - i.e., the state obtained from Eqs. (2) by creating a charged exciton, $|x\rangle$. The effective
Hamiltonian in the three level sub-space is:

$$
H_{0}=-\Delta|x\rangle\langle x|+\left(\frac{\Omega}{2}|1\rangle\langle x|+\text { h.c. }\right),
$$

where $\Delta$ is the detuning from the $|1\rangle \rightarrow|x\rangle$ transition. The detuning also includes a part which is due to Coulomb interactions present in the trion state. The Coulomb interactions present in the trion state modify the "bare" trion energy $\epsilon_{0,1 / 2}^{e}+\epsilon_{0,-1 / 2}^{e}+$ $\epsilon_{0, \sigma_{h}}^{h}$. The interaction $H^{c c}$ changes the bare state $c_{0,+1 / 2}^{\dagger} c_{0,-1 / 2}^{\dagger} h_{0, \sigma_{h}}^{\dagger}|\mathrm{vac}\rangle$ into the physical interacting state $\left|x, \sigma_{h}\right\rangle$, where $\sigma_{h}$ is the total angular momentum projection of the hole state. Such states were observed and studied experimentally in single self-assembled QDs [16]. This three-level subspace, spin selectively coupled to a laser field, was also the basis for a quantum implementation scheme which we previously proposed [4]. For further details on obtaining the this effective Hamiltonian and their single particle states, the reader is referred to [17].

\section{E. Coulomb interaction}

With regards to the carrier-carrier interaction part of the Hamiltonian, Eq. (1), it should be noted that, as opposed to higher dimensional quantum structures, in QDs carrier-carrier interactions only induce an energy level renormalization without causing scattering or dephasing. The carrier-carrier interaction is small, its smallness being expressed by the parameter $L / a_{B}$, where $L$ is the typical spatial dimension of the QD and $a_{B}$ is the effective Bohr radius.

\section{F. Incoherent part: exciton recombination}

The trion state contains two electrons and a hole, an electron and the hole can recombine and emit a photon. The radiative recombination rates are calculated by the Fermi Golden Rule under the dipole approximation. In 
this approximation the recombination is controlled by the dipole matrix element defined by: $\langle f|\epsilon \cdot \mathbf{p}| i\rangle$ where $|i\rangle$ and $|f\rangle$ are the initial and final states respectively. The possible recombination channels are determined by symmetry considerations regarding the dipole matrix element. Using the Bloch functions to represent the states one can see that the only non-vanishing transitions are $\langle S|x| X\rangle,\langle S|y| Y\rangle,\langle S|z| Z\rangle$. Due to the symmetry of the QD we assume $\langle S|x| X\rangle \sim\langle S|y| Y\rangle$ but the ratio of these matrix elements to $\langle S|z| Z\rangle$ depends on many factors, e.g. QD shape and size.

From the above considerations we see that $\mathrm{HH}$ transitions only occur via emission of polarized light. Without the mixing of the hole states an, electron-hole pair composing the trion state can only recombine emitting a $\sigma^{+}$polarized photon. When there is mixing of the $\mathrm{HH}$ and LH states, there is a further decay channel via the emission of a linear polarized photon, $\sigma^{0}$. This can be seen by considering the following matrix elements: $\left\langle\frac{1}{2}, \frac{1}{2}|z| \frac{3}{2}, \frac{1}{2}\right\rangle \sim\langle S|z| Z\rangle,\left\langle\frac{1}{2},-\frac{1}{2}|z| \frac{3}{2},-\frac{1}{2}\right\rangle \sim\langle S|z| Z\rangle$.

\section{STATE READ-OUT BY QUANTUM JUMPS}

Our proposed measurement scheme is based on shining a continuous $\sigma^{+}$polarized laser pulse in resonance with the trion excitation on a QD containing an electron in the ground state. Continuously monitoring of the luminescence from the the QD we expect to see photons emitted from the QD, i.e. fluorescence, if the initial spin state of the excess electron in the QD corresponds to $|1\rangle$. If instead the excess electron is initially in the spin state corresponding to $|0\rangle$, we expect to see no photons, i.e., dark periods. This measurement scheme belongs to the category of indirect measurements [18] which are composed of a two-step process: first the system, corresponding to the confined electron in the above scheme, is brought in contact with a "probe" quantum system prepared in an initial state, which corresponds to the laser field prepared with a given polarization. The second step is the direct measurement of some observable of the probe which in the above scheme simply means measuring the emitted photon. Our proposed measurement scheme follows the requirements from a high precision indirect measurement: First, the direct measurement of the quantum probe, (the photon) does not begin before the first step is complete, (the photon carrier interaction is over). Second (in the non-mixing case which is defined later on), the measurement of the probe observable does not contribute significantly to the total measurement error, i.e., we have a high ability to detect the emitted photon. Having met the above requirements, the only source of error in our measurement scheme arises from the internal uncertainties of the quantum probe, e.g., the exact polarization of the laser pulse. In the following we will assume that the laser pulse can be shined in a given direction and with an exact polarization. We shall also assume in the next sections perfect photon detection, i.e. that every photon emitted from the QD is detected with probability 1 which we describe by a detection efficiency $\eta=1$. The latter of course is an unrealistic limit and we will discuss in detail what happens when the photon detection is less then 1. Using an avalanche photo-diode detector the detection efficiency is about $80 \%$ (for example see [19]). The typical wave length emitted by the recombination process in the QDs lies well within the spectral window which is due to the cutoff by band gap energy of such detectors. The main source for low detection efficiency is due to the probability for the emitted photon to reach the detector, i.e. the difficulty arising due to finite angle coverage of the detector. The situation however can be significantly improved by coupling the QD with a microcavity as described in [8].

The measurement scheme is theoretically described by 
the quantum-jump technique. Employing this technique we write down an effective non-Hermitian Hamiltonian describing the dynamics of a reduced density matrix $\rho$. The typical time associated with this measurement scheme is the time of measurement $T_{M}$ which is the time the laser pulse is switched on. We choose the measurement time to be such that it minimizes the measurement error which is a sum of two factors: the first is the possibility that starting with an electron in state $|1\rangle$ still during the whole measurement time no photon was detected from the QD. We denote the probability for such an error by $E r_{1}(t)$. The second kind of error, denoted by $E r_{2}(t)$, is that starting the system off in state $|0\rangle$ a photon was emitted (and detected) from the QD. $T_{M}$ is chosen so that it minimizes the sum of $\operatorname{Er}_{1}(t)+E r_{2}(t)$. We start by describing the measurement process in the case with no $\mathrm{HH}$, LH mixing.

\section{A. No mixing scenario}

Cases with no HH LH mixing have been experimentally observed in InP QDs [20] as well as in resonant excitation experiments [21], [22] for InAs/GaAS which showed that carrier spins are totally frozen on exciton life time scale. The case with no mixing is defined by $\varepsilon=0$, were $\varepsilon$ is the mixing parameter to be defined later. Shining a $\sigma^{+}$pulse on the QD we obtain due to the Pauli blocking effect in QDs the usual two-level situation: no fluorescence from initial state $|0\rangle$, full fluorescence from state $|1\rangle$. Assuming perfect photon detection $\eta=1$, one measurement is a one shot measurement, i.e. one has to wait long enough to ensure that if starting with the qubit in state $|1\rangle$ a single photon will be emitted, but once this photon is emitted the system is known to be in state $|1\rangle$. If $\eta<1$ one has to wait long enough to make sure a detected photon will be observed, that is, if the electron was in state $|1\rangle$ one has to wait long enough to ensure enough photons will be emitted so as at least one of them will be detected.

The limiting process which determines the errors $E r_{1}$ and $E r_{2}$ described above is the spin coherence time in the QD. Therefore in this case the measurement time, $T_{M}$, is controlled by the typical time in which a spin flip transition occurs in the QD. The issue of spin dephasing times in QDs is still unresolved, the reason being that experimental verification of theoretical estimates is still dearly needed. Moreover the spin dephasing time strongly depends on the size and shape of the QD. Theoretical estimates range from 1-10 ns [23] to smaller than $\mu \mathrm{s}[25]$.

In the following we shall assume the spin flip rate to be in the $\mu$ s range. A detailed treatment of the recycling based measurement scheme for the case of spin relaxation of the order of ns was given by Shabeav et. al. [10] who showed that the spin relaxation is suppressed by coupling to light in the strong coupling regime, which can be viewed as a sort of quantum Zeno like effect.

The fluorescence pattern is governed by [24]

$$
\kappa_{r e c} \tilde{\rho}_{x x}^{(0)}\left(t \mid t_{0}\right)=-\frac{d}{d t}\left[\tilde{\rho}_{11}^{(0)}\left(t \mid t_{0}\right)+\tilde{\rho}_{x x}^{(0)}\left(t \mid t_{0}\right)\right]
$$

where $\tilde{\rho}^{(0)}$ is the reduced density matrix for a two level system, $|1\rangle,|x\rangle$ in the sub-space where no photons have been emitted since $t_{0}$ and we estimate the recombination rate $\kappa_{\text {rec }} \approx 10^{9} \mathrm{sec}^{-1}$. Eq (7) describes the decay of the trace of $\tilde{\rho}$, i.e., the probability to emit a photon. One continues to obtain photons (or no photo-emission) as long as the original spin state of the electron has not been flipped. Considering the typical time for a spin flip to be in the $\mu$ s range [25] the average number of photons emitted in a fluorescence pattern, which is given by the ratio of the spin coherence time to the typical rate for spontaneous emission, should be of the order of $10^{3}$. In this the detection efficiency can be made greater 
by using detectors which do not need to detect singlephoton events [19].

\section{B. Case with mixing}

Even though in recent experiments in $\mathrm{InAs} / \mathrm{GaAs}$ QDs [21], [22] exhibit no decay of both linear and circular luminscence polarization, indicating that there is apparently no HH LH mixing in these QDs, typically self-assembled QDs will exhibit mixing of the HH and LH states. Such mixing invalidates the assumption of perfect Pauli blocking with $\sigma^{+}$light and can be viewed as a rotation of the basis by an angle $-\varepsilon$ in the $\{|0\rangle,|1\rangle\}$ space. The mixing parameter $\varepsilon$, depends on the material and the sample, and can reach values up to the order of $10 \%$ which is the value we employ for our calculations.

Introducing mixing requires one to treat the full threelevel lambda configuration shown in Fig. 2 As opposed to the usual atomic lambda configuration [26], here one can not distinguish between the $|0\rangle\langle x|$ and $|1\rangle\langle x|$ transitions. These two transitions are mediated through the same photon. This is basically the signature of the back action of the photon on the measured spin of the electron.

Employing the quantum-jump technique the dissipative evolution of the density matrix $\tilde{\rho}(t)$, in the case were the photon is a $\sigma^{+}$light pulse shined in the growth direction, is given by (see, e.g.,[27])

$$
\begin{aligned}
& \dot{\tilde{\rho}}_{00}=i \frac{\Omega}{2} \varepsilon\left(\tilde{\rho}_{x 0}-\tilde{\rho}_{0 x}\right) \\
& \dot{\tilde{\rho}}_{11}=i \frac{\Omega}{2}\left(\tilde{\rho}_{x 1}-\tilde{\rho}_{1 x}\right) \\
& \dot{\tilde{\rho}}_{x x}=i \frac{\Omega}{2}\left[\tilde{\rho}_{1 x}-\tilde{\rho}_{x 1}+\varepsilon\left(\tilde{\rho}_{0 x}-\tilde{\rho}_{x 0}\right)\right]-\left(1+\tilde{\varepsilon}^{2}\right) \kappa \tilde{\rho}_{x x} \\
& \dot{\tilde{\rho}}_{01}=i \frac{\Omega}{2}\left(\varepsilon \tilde{\rho}_{x 1}-\tilde{\rho}_{0 x}\right) \\
& \dot{\tilde{\rho}}_{0 x}=i \frac{\Omega}{2}\left[\varepsilon\left(\tilde{\rho}_{x x}-\tilde{\rho}_{00}\right)-\tilde{\rho}_{01}\right]-\left(1+\tilde{\varepsilon}^{2}\right) \frac{\kappa}{2} \tilde{\rho}_{0 x} \\
& \dot{\tilde{\rho}}_{1 x}=i \frac{\Omega}{2}\left(\tilde{\rho}_{x x}-\tilde{\rho}_{11}-\varepsilon \tilde{\rho}_{10}\right)-\left(1+\tilde{\varepsilon}^{2}\right) \frac{\kappa}{2} \tilde{\rho}_{1 x}
\end{aligned}
$$

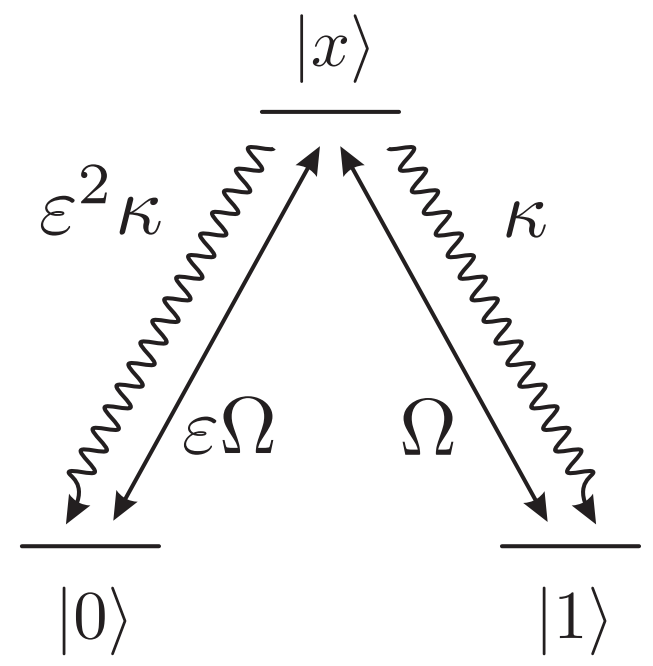

Fig. 2. The lambda configuration one has to consider to include hole mixing.

where $\tilde{\varepsilon}^{2}=\varepsilon^{2}+\varepsilon^{\prime 2}$ and $\varepsilon^{\prime}$ is a result of a further recombination channel, described below, which is allowed due to the mixing. Introducing mixing of the HH and LH states affects both the coherent and the incoherent (recombination) part of the Hamiltonian in different ways. In the case where there is a single $\sigma^{+}$ light pulse shined in the growth direction, the ratio of the Rabi frequencies for the $|1\rangle\langle x|$ and $|0\rangle\langle x|$ transitions is given by $\varepsilon$, but the incoherent recombination rates are not simply given by $\varepsilon^{2}$. The reason for this is that a further recombination channel is "opened up" due to mixing. The allowed recombination transitions are restricted by allowed photon emitted states which should have a total angular momentum of one. Therefore there are two possibilities for such a recombination process (see Se凹II-F. Since the second decay channel is again proportional to the mixing parameter squared, $\varepsilon^{2}$, but (8) with a different coefficient determined by the dipole matrix element $\langle S|z| Z\rangle$, we describe its effect on the decay by adding to $\varepsilon^{2}$ a further $\varepsilon^{\prime 2}$ term. In a previous paper [17] we neglected this further decay channel here 


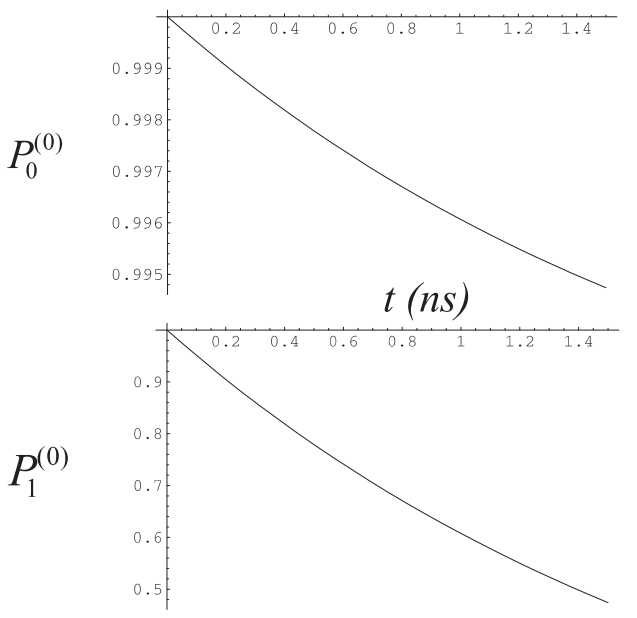

Fig. 3. Probability that at time $t$ the first photon has not yet been emitted, starting from state $|0\rangle$ (above) or $|1\rangle$ (below) at time $t=0$. Parameters are quoted in the text.

we considered both cases: $\varepsilon^{\prime}=0$ and the opposite limit $\varepsilon^{\prime} \sim \varepsilon$ in which we take $\tilde{\varepsilon}=\sqrt{2} \varepsilon$.

The probability that at time $t$ no photon has been emitted, starting from state $\alpha$ at time $t_{0}$, is

$$
P_{\alpha}^{(0)}\left(t-t_{0}\right)=\operatorname{tr}[\tilde{\rho}(\alpha, t)]
$$

where at the initial time $t_{0}$ we take $\tilde{\rho}\left(\alpha, t_{0}\right) \equiv|\alpha\rangle\langle\alpha|$. Fig. 3 shows an example of their evaluation with $\Omega=3$ $\mathrm{meV}, \kappa=1 \mathrm{~ns}^{-1}$ and $\varepsilon=\tilde{\varepsilon}=0.1$. For comparison we took the case (same parameters) in which $\frac{\tilde{\varepsilon}}{\sqrt{2}}=\varepsilon=$ 0.1 . In this case we got exactly the same results as in Fig. 3 As can be seen from Eq. Eq. (8) the $\tilde{\varepsilon}^{2}$ term can be neglected compared to 1 . It is also worthwhile to notice that $P_{1}^{(0)}(t)$ does not decay to zero as in the case $\varepsilon=0$ rather both $P_{1}^{(0)}$ and $P_{0}^{(0)}$ decay to some constant value asymptotically since states $|0\rangle$ and $|1\rangle$ no longer correspond to the "dark" and "fluorescent" states respectively, i.e. there is also a "dark side" to state $|1\rangle$.

There are a few major differences in the above case with mixing in contrast to the "common" lambda configuration [26], commonly used in quantum optics. In Eq. (8) both of the recycling terms, $\kappa \tilde{\rho}_{x x}$ and $\varepsilon^{2} \kappa \tilde{\rho}_{x x}$, are missing, since it is the same photon that induces both these transitions, i.e. we can not distinguish between the two transitions via photon detection. This implies that, when the first photon is emitted, say at time $t_{1}$, the system collapses either into state $|0\rangle$ - with probability $p_{0}=\tilde{\varepsilon}^{2} /\left(1+\tilde{\varepsilon}^{2}\right)$ - or into state $|1\rangle$ - with probability $p_{1}=1 /\left(1+\tilde{\varepsilon}^{2}\right)-$, whence the evolution starts over again. Therefore the probability that, at the time $t>t_{i}$ ( $i \geq 1)$, the $(i+1)$-th photon has not been emitted, is

$$
P_{\alpha}^{(i)}\left(t-t_{i}\right)=\frac{\tilde{\varepsilon}^{2} P_{0}^{(0)}\left(t-t_{i}\right)+P_{1}^{(0)}\left(t-t_{i}\right)}{1+\tilde{\varepsilon}^{2}},
$$

which is independent of the initial state $|\alpha\rangle$. The possibility for the emitted photon to induce a flip adds to the back-action of the quantum "probe" on the measured system. The measurement time is again limited by the time the life time the quantum "memory" ,i.e., by the typical time a spin flip will occur. But in this case the spin flip will occur with high probability due to repeated photon emissions. The measurement time is therefore back-action limited. A typical photo-emission pattern will look like Fig. 4 a sequence of pulses, each one made out of a bunch of the order of $1 / \tilde{\varepsilon}^{2}$ photons, separated by no-emission windows. This is the typical quantumjump pattern one obtains in the presence of an emission probability having the form of a sum of different exponentials like Eq. 10. $N$, the average number of photons in the first bunch, is given by a random walk like calculation: $N=\sum_{n=0}^{\infty} n q^{n} p=q \frac{\partial}{\partial q} \sum_{n=0}^{\infty} q^{n} p$, where $q=1 /\left(1+\tilde{\varepsilon}^{2}\right)$ and $p=1-q$ stand for the probabilities to decay into states $|1\rangle$ and $|0\rangle$ respectively. Thus one can easily see $N \approx \tilde{\varepsilon}^{-2}$. In our case as discussed above (see Fig. 4 it is of the order of $\tilde{\varepsilon}^{-2}=50$. It is only through this first bunch of photons, which are emitted almost immediately in the case of state $|1\rangle$, and after a sensible delay in the case of state $|0\rangle$, that one can discriminate the two patterns. 
$|0\rangle$

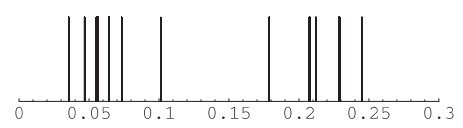

$t(m s)$

$|1\rangle$
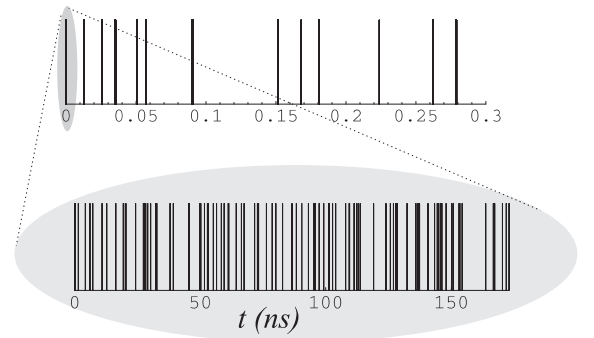

Fig. 4. Simulation of photon counts for a system starting from state $|0\rangle$ (top) and from state $|1\rangle$ (middle). An expanded view of the first few photon counts is displayed in the bottom graph. Parameters are the same as in Fig. 3

Starting with the system in state $|1\rangle$ there is a possibility for no photon to be emitted from the QD during the whole measurement time. The probability for this type of error is given by $E r_{1}(t)=P_{1}^{(0)}(t)$. In the other case starting with the system initially in $|0\rangle$ at least one photon might be emitted during the measurement time. The probability for this sort of error is given by: $E r_{2}(t)=1-P_{0}^{(0)}(t)$. The measurement time has to be chosen in such a way as to minimize the sum of these two errors. For the same parameters employed in Fig. 3 we obtain an estimate for the optimal measurement time of the order of a few tens of ns. What typically happens in practice is that, as shown in Fig. 4. by appropriate time windowing the first bunch of photons coming from state $|1\rangle$ can be safely discriminated from the (later) photons coming from state $|0\rangle$.

\section{Two laser pulses}

One can try to improve the detection capability by shining more than one laser pulse on the QD. Shining a further laser pulse, phase matched to the first, in the in-plane direction with a $\hat{z}$ linear polarization, $\sigma^{0}$, couples only to the $\mathrm{LH}$ wave function since only they posses a $|Z\rangle$ Bloch function component. There are two Rabi frequencies in this case: $\Omega$ for the $\sigma^{+}$ polarized light shined in the growth direction $\hat{z}$ and $\Omega^{\prime}$ for the linear polarized laser pulse (in the $\hat{z}$ direction) shined in the in-plane direction, e.g. $\hat{x}$. The modified equations of motion can be obtained from Eq(9) by modifying the coherent part of the Hamiltonian, i.e., the Rabi frequencies. The modified Rabi frequencies are: $\Omega \rightarrow \Omega \pm \varepsilon \Omega^{\prime}$ and $\varepsilon \Omega \rightarrow \varepsilon\left(\Omega \pm \Omega^{\prime}\right)$. This can be seen by considering the following matrix elements: $\left\langle\frac{1}{2}, \frac{1}{2}|z| \frac{3}{2}, \frac{1}{2}\right\rangle \sim\langle S|z| Z\rangle,\left\langle\frac{1}{2},-\frac{1}{2}|z| \frac{3}{2},-\frac{1}{2}\right\rangle \sim\langle S|z| Z\rangle$, which show that the LH part of the mixed HH LH state will couple to the conduction band state. The Rabi frequency for the coupling being $\Omega^{\prime}$ and $\varepsilon$ is the LH "part" of the mixed state which is coupled.

Since the further laser pulse modifies only the coherent part of the Hamiltonian it will affect the measurement efficiency through the change of the time of measurement and the value of $\operatorname{Er}_{2}\left(T_{M}\right)$. The bottleneck process limiting the measurement time $T_{M}$ is the finite probability $p_{0}=\tilde{\varepsilon}^{2} /\left(1+\tilde{\varepsilon}^{2}\right)$, for decay into state $|0\rangle$ every time a photon is emitted. This finite probability is controlled by the decay rate, i.e., the incoherent part of the Hamiltonian, and as such will not be affected by the second lase pulse. Thus the first bunch of photons emitted from the initial state $|1\rangle$ will be of the order of $1 / \tilde{\varepsilon}^{2}$ photons, independent on the number of laser pulses. The measurement time, $T_{M}$ is affected by the coherent part of the Hamiltonian since it determines how quickly a photon will be emitted when the system starts off in state $|1\rangle$. The error rate $\operatorname{Er}_{2}\left(T_{M}\right)$ is also affected by the coherent part. Therefore one can see the optimal case will be achieved for $\Omega=-\Omega^{\prime}$. 


\section{Finite detection efficiency}

We now consider the case in which $\eta<1$. For this case there is a further measurement error denoted by $E r_{3}(t)$. This error is due to the possibility that starting off initially in state $|1\rangle$ the QD can emit a photon/photons which will go undetected and the spin can flip into state $|0\rangle$, i.e., the information regarding the spin state is lost without being detected. The probability for such an error is given by

$$
\begin{aligned}
E r_{3} & =\tilde{\varepsilon}^{2} \sum_{n=0}^{N}\left(\frac{1-\eta}{1+\tilde{\varepsilon}^{2}}\right)^{n+1} \\
& =\frac{\tilde{\varepsilon}^{2}(1-\eta)}{\tilde{\varepsilon}^{2}+\eta}\left[1-\left(\frac{1-\eta}{1+\tilde{\varepsilon}^{2}}\right)^{N+1}\right],
\end{aligned}
$$

which is simply the sum over $n$ incidents in which the emitted photons were not detected and no spin-flip occurred and on the $n+1$ incident such a spin flip occurred (without the photon being detected). Taking a relatively low detection efficiency $\eta=0.8$ we obtain an error due to finite detection efficiency less than $0.5 \%$.

Working with a detector with a finite efficiency means that we have to choose the measurement time so as to ensure the fluorescent state emits a few photons thus increasing the probability one of them will be detected. This will also increase the probability for an error due to a photon being emitted by the initial state $|0\rangle$ since $P_{0}^{(0)}(t)$ decays exponentially with time, but the main error source are spin flips after undetected photons.

\section{CONCLUSION}

Employing a quantum-jump technique, we have theoretically demonstrated that it is possible, utilizing the resonance-luminescence technique, which has been proven to be very effective in the field of quantum optics, to measure the spin of an excess electron confined to a QD. The complications arising from the underlying crystal have been critically examined and we have shown that even in the presence of heavy and light hole mixing one is still able to measure the electron's spin to a very high degree of accuracy.

\section{REFERENCES}

[1] L. Jacak and P. Hawrylak and Wojs, Quantum Dots Springer, Berlin: Addison-Wesley, 1998.

[2] J. H. Reina, L. Quiroga and N. F. Johnson, Phys. Rev. A 62, 012305 (2000); F. Troiani, U. Hohenester, E. Molinari, Phys. Rev. B 62, R2263 (2000); it ibid, Phys. Stat. Sol. B 224, 849 (2001); E. Biolatti et al., Phys. Rev. Lett. 85, 5647 (2000); Phys. Rev. B 65, 075306 (2002).

[3] D. Loss and D. P. DiVincenzo, Phys. Rev. A 57, 120 (1998); X. Hu and S. Das Sarma, Phys. Rev. A 61, 062301 (2000); ibid, 64, 042312 (2001); M. Bayer, P. Hawrylak, K. Hinzer, S. Fafard, M. Korkusinski, Z. R. Wasilewski, O. Stern and A. Forchel, Science 291, 451 (2001);

[4] E. Pazy, T. Calarco, I. D’Amico, P. Zanardi, F. Rossi and P. Zoller, Euro. Phys. Lett. 62, 175 (2003); Journal of Superconductivity: Incorporating Novel Magnetism 16 , 383 (2003).

[5] H. -S. Goan and G. J. Milburn, Phys. Rev B 64, 235307 (2001).

[6] E. Pazy, I. D’Amico, P. Zanardi and F. Rossi Phys. Rev.B. 64, 195320 (2001); Physica B: Physics of Condensed Matter 314, 20 (2002).

[7] U. Hohenester, Solid State Commun. 118, 151 (2001).

[8] A. Imamoğlu, Fortschr. Phys. 48, 987 (2000).

[9] S. Cortez, O.Krebs, S. Laurent, M. Senes, X. Marie, P. Voisin, R. Ferreira, G. Bastard, J. M. Gérard and T. Amand, Phys. Rev. Lett. 89, 207401-1 (2002).

[10] A. Shabeav, Al. L. Efros, D. Gammon and I.A. Merkulov cond-mat/0307369

[11] R. J. Warburton, C. S. Dürr, K. Karrai, J.P. Kotthaus, G. Medeiros-Ribeiro and P. M. Petroff, Phys. Rev. Lett. 79, 5282 (1997); V. K. Kalevich, M. Paillard, K. V. Kavokin, X. Marie, A. R. Kovsh, T. Amand, A. E. Zhukov, Yu. G. Musikhin, V. M. Ustinov, E. Vanelle and B. P. Zakharchenya, Phys. Rev. B. 64, 045309 (2001). Gang Chen, N. H. Bonadeo, D. G. Steel, D. Gammon, D. S. Katzer, D. Park and L. J. Sham, Science 289, 1906 (2000).

[12] P. Y. Yu and M. Cardona, Fundamentals of Semiconductores , Berlin: Springer, 2001.

[13] E. O. Kane in editors R. K. willardson and A. C. Beer editors Semiconductores and Semimetals vol 39. , New-York:Academic , 1966. 
[14] G. Bastard, Wave Mechanics Applied to Semiconductores Hetrostructures Les Ulis Cedex,France : Editions de physique, 1988.

[15] A.J. Williamson, L. W. Wang and A. Zunger Phys. Rev B 62, 12963 (2000).

[16] A.S. Bracker, J.G. Tischler, V. I. Korenev and D. Gammon, Phys. Stat. Sol. 238, 266 (2003).

[17] T. Calarco, A. Datta, P. Fedichev, E. Pazy, P. Zoller, Phys. Rev. A 68, 012310 (2003).

[18] V. B. Braginsky and F. Y. Khalli, Quantum Measurement Great Britain: Cambridge University Press, 1992.

[19] H.-A. Bachor A Guide to Experiments in Quantum Optics (Wiley-VCH, Weinheim, 1998).

[20] L. Langof, E. Ehrenfreund, E.Lifshitz, O. I.Micic and A. J. Nozik J. Phys. Chem. B 106, 1606 (2002).

[21] M. Paillard, X. Marie, P. Renucci, T. Amand, A. Jbeli and J. M. Gérard Phys. Rev. Lett. 86, 1634 (2001).

[22] A. S. Lenihan, M.V. Gurudev Dutt, D. G. Steel, S.Gosh and P. K. Bhattacharya Phys. Rev. Lett. 88, 223601 (2002).

[23] I. A. Merkulov, Al. L. Efros and M. Rosen Rev. B 65, 205309 (2002).

[24] P. Zoller et al., Phys. Rev. A 35, 198 (1986).

[25] A. V. Khaetskii and Y. V. Nazarov Phys. Rev. B 61, 12639 (2000).

[26] P. Zoller, M. Marte, D.F. Walls, Phys. Rev. A 35, 198 (1987).

[27] C. W. Gardiner and P. Zoller Quantum Noise, 2nd ed. Springer, 2000. 